\title{
Reflets
}

Revue ontaroise d'intervention sociale et communautaire

\section{Vivre intensément l'humain : une exploration du deuil après le VIH-sida}

\section{Susan Cadell et Dennis Haubrich}

Volume 12, numéro 1, 2006

Spiritualité et intervention sociale

URI : https://id.erudit.org/iderudit/013441ar

DOI : https://doi.org/10.7202/013441ar

Aller au sommaire du numéro

Éditeur(s)

Reflets : Revue ontaroise d'intervention sociale et communautaire

ISSN

1203-4576 (imprimé)

1712-8498 (numérique)

Découvrir la revue

Citer cet article

Cadell, S. \& Haubrich, D. (2006). Vivre intensément l'humain : une exploration du deuil après le VIH-sida. Reflets, 12(1), 127-143.

https://doi.org/10.7202/013441ar

Tous droits réservés (C) Reflets : Revue ontaroise d'intervention sociale et communautaire, 2006
Ce document est protégé par la loi sur le droit d'auteur. L'utilisation des services d'Érudit (y compris la reproduction) est assujettie à sa politique d'utilisation que vous pouvez consulter en ligne.

https://apropos.erudit.org/fr/usagers/politique-dutilisation/ 


\title{
Vivre intensément l'humain : une exploration du deuil après le VIH-sida ${ }^{1}$
}

\author{
Susan Cadell, \\ School of Social Work E Family Studies, University of British Columbia \\ et
}

\section{Dennis Haubrich,}

School of Social Work, Ryerson University

Cette étude a pour but d'explorer l'expérience d'individus ayant pris soin de personnes décédées des complications dues au VIHsida. Des questions ouvertes ont permis d'analyser les expériences positives aussi bien que négatives de quinze personnes interviewées. Considérée comme ressource potentielle, la spiritualité faisait partie des sujets abordés dans cette étude. Les expériences positives prennent parfois racine dans la spiritualité et la religion. La spiritualité dans le deuil était très importante pour les soignants.

\section{Introduction}

L'épidémie du sida croît d'année en année, entrânant avec elle un nombre grandissant d'individus ayant pris soin de personnes qui en sont atteintes ou en sont décédées. En plus de subir le stress du deuil dans le contexte du VIH-sida, plusieurs de ces personnes se trouvent stigmatisées par le fait qu'elles sont ellesmêmes séropositives. La réprobation sociale de leurs relations leur occasionne des pertes considérables et continues, même après la mort de l'être cher. De surcroit, il leur est fréquent de vivre des deuils successifs. 
"La plupart des études sur le sida portent sur ses aspects négatifs. Ses effets alarmants sont bien documentés. »

\section{"L'hétérosexisme} et l'homophobie peuvent ajouter à la stigmatisation aussi bien qu'à une augmentation $d u$ stress. ”
La plupart des études sur le sida portent sur ses aspects négatifs. Ses effets alarmants sont bien documentés. En Amérique du Nord, l'épidémie se concentre dans les communautés lesbienne, gaie, bisexuelle et transsexuelle, où l'on constate un taux élevé de décès. Il n'est pas inhabituel pour des hommes gais d'avoir perdu des dizaines d'amis et de connaissances (Shernoff 1995, 1997b). Le nombre moyen de pertes cité par plusieurs études américaines varie entre 8 et 67,7 (Nord 1996a). Les pertes sont telles que la mort est chose habituelle dans la communauté gaie (Goodkin, Blaney,Tuttle et al. 1996; Neugebauer, Rabkin,Williams, Remien, Goetz \& Gorman 1992) et que le deuil représente une épidémie parallèle à celle du sida (Wardlaw 1994). Les pertes incessantes associées au VIH et au sida sont une source de traumatismes. (Nord 1996a, 1996b, 1997, 1998; Shernoff 1997a). Les personnes ayant subi des pertes multiples vivent un traumatisme (Bigelow \& Hollinger 1996; Cadell 2001, 2003; Cadell, Regehr \& Hemsworth 2003; Gluhoski, Fishman \& Perry 1997a, 1997b; Goodkin et al. 1996; Houseman \& Pheifer 1988; Martin \& Dean 1993; Sikkema, Kalichman, Kelly \& Koob 1995). L'hétérosexisme et l'homophobie peuvent ajouter à la stigmatisation aussi bien qu'à une augmentation du stress.

Se tourner vers la religion peut être un moyen de combattre ce stress (Pargament \& Park 1995). Souvent, les concepts de religion et de spiritualité sont utilisés de façons interchangeables. Il y a cependant entre les deux des aspects distinctifs. La religion réfere à une structure ou à une organisation qui définissent les croyances d'un groupe d'individus. Canda et Furman (1999) la définissent comme un système organisé de croyances, de rites et de symboles ayant un double objectif : faciliter l'approche vers le transcendant ou le sacré et promouvoir la compréhension et la responsabilité entre les membres d'une communauté. Kozeny (2004) voit la religion comme un système de croyances et de normes sociales qui expose les personnes à des idées et à des expériences spirituelles en plus de les aider à incorporer des valeurs à leur vie quotidienne.

Si la religion est définie en termes de structure, de système et d'organisation, les définitions de la spiritualité suggèrent un rapport 
"Dans les communautés lesbienne et gaie, beaucoup de personnes se sont éloignées de la religion." plus personnalisé, lié davantage aux croyances individuelles. Canda et Furman (1999) définissent la spiritualité comme "une quête " visant à comprendre les réponses aux questions ultimes de la vie, du sens de la vie et de la relation avec le sacré. Hardy (1982) propose, comme définition de la spiritualité, un état d'esprit qui libère la personne de son isolement. Les êtres humains cherchent à créer avec d'autres personnes des liens leur permettant de s'épanouir.

Ces définitions amènent à distinguer religion et spiritualité. Bien que le concept de la spiritualité ait ses origines dans la religion (Jacobs 1997), il a pris depuis plus d'ampleur. Cependant, il est clair qu'il existe un lien important entre les deux concepts. Kozeny (2004) indique que la religion peut être l'objectif à travers lequel la spiritualité se rend visible. La religion peut offrir une structure ou une organisation à ceux qui partagent des notions communes de spiritualité. Cette dernière, quant à elle, semble indépendante, dans le sens qu'elle peut exister tant à l'extérieur qu'à l'intérieur de la religion.

La religion peut offrir une feuille de route vers la destination ultime (Pargament \& Park 1995) ce qui, dans bien des cas, ne permet pas à une personne lesbienne, gaie ou transsexuelle de s'accepter en tant que telle (Helminiak 1995). Dans les communautés lesbienne et gaie, beaucoup de personnes se sont éloignées de la religion (Hardy 1998).

Malgré la forte probabilité de stress dans le deuil, peu de recherches ont exploré l'expérience de ceux et de celles qui ont vécu la mort d'un être cher pour cause de sida. Encore moins d'études sont consacrées à la spiritualité dans la vie des soignants.

Une étude longitudinale effectuée à San Francisco et s'étalant sur une période de sept années (Folkman, Chesney, Collette, Boccellari, \& Cooke 1996; Folkman \& Moskowitz 2000; Folkman, Moskowitz, Ozer, \& Park 1997; Moskowitz, Folkman, Collette, \& Vittinghoff 1996; Wrubel \& Folkman 1997) a permis la cueillette de données quantitatives et qualitatives de la part d'hommes gais, engagés dans des relations à long terme, qui prenaient soin de leur partenaire atteint du sida. 
"Le cadre théorique de cette recherche est l'approche transactionnelle $d u$ stress et de l'adaptation... Cette approche propose que le stress vécu par l'individu soit le résultat de sa façon d'évaluer la situation et que les stratégies d'adaptation soient le résultat de la transaction entre les ressources personnelles de l'individu et son environnement.»
Les soignants dont le partenaire est mort pendant l'étude ont été interviewés subséquemment (Richards \& Folkman 1997). La spiritualité a émergé comme un mécanisme important dans l'adaptation. Trois ou quatre ans après la mort, la spiritualité restait importante, mais son rôle dans la vie de chaque soignant avait changé (Richards, Acree \& Folkman 1999). Les chercheurs reconnaissent les limites de leur étude : les questions ne portaient pas directement sur la spiritualité. Dans le cas des soignants qui n'en ont pas parlé, il n'y a aucun moyen de déterminer si c'est par manque de spiritualité ou par simple refus de leur part.

Le cadre théorique de cette recherche est l'approche transactionnelle du stress et de l'adaptation (Lazarus \& Folkman 1984; Folkman 1997). Cette approche propose que le stress vécu par l'individu soit le résultat de sa façon d'évaluer la situation et que les stratégies d'adaptation soient le résultat de la transaction entre les ressources personnelles de l'individu et son environnement. Dans le premier modèle de l'approche transactionnelle du stress et de l'adaptation proposée par Lazarus et Folkman (1984), les émotions positives n'étaient possibles qu'en cas de résolution favorable de la situation. Folkman (1997) a proposé un nouveau modèle qui envisage la possibilité d'émotions positives, même en cas d'échec dans la résolution de la situation.

Notre recherche a été conçue pour adresser spécifiquement la spiritualité parmi d'autres facteurs (soutien social, stress, etc.) qui influencent le vécu des soignants après la mort d'un être cher. En posant à chacun des participants la question «Est-ce que la spiritualité vous a aidé [dans le processus du changement après la mort de quelqu'un au sida]? " — voir en annexe le guide d'entretien - aucune définition de la spiritualité n'était donnée. Chaque individu était libre de la définir à sa façon. 


\section{La méthodologie}

\section{Les participants}

Les participants à cette recherche qualitative ont été choisis à partir d'une étude quantitative (Cadell 2001; 2003; Cadell, Regehr \& Hemsworth 2003). Pour répondre au questionnaire de l'étude quantitative, chaque participant devait avoir pris soin de quelqu'un qui est mort des causes liées au VIH-sida. Les participants ont été recrutés à l'aide d'affiches dans des lieux publics, auprès d'organismes voués au sida, lors d'événements de la fierté gaie et de conférences sur le sida.

Pour participer, chaque personne laissait un message à un numéro sans frais. L'étude leur a été expliquée et, avec leur accord, le questionnaire leur a été envoyé. Chaque questionnaire demandait à la personne si elle acceptait de prendre part à une entrevue. Les participants ayant acquiescé à l'invitation ont été invités à des entrevues se déroulant dans quatre grandes villes canadiennes.

\section{Protocole d'interview}

Les interviews étaient organisées autour d'une liste de questions, laquelle était conforme à la littérature portant sur les soignants et sur le deuil dans le contexte du VIH-sida (Folkman, Chesney, Collette, Boccellari \& Cooke 1996; Folkman, Chesney, Cooke, Boccellari \& Collette 1994; Folkman, Moskowitz, Ozer \& Park 1997; Richards, Acree \& Folkman 1999). Chaque interview commençait par une invitation à parler de la personne décédée et se poursuivait par des questions sur la façon dont la vie des soignants a été changée par l'expérience de soigner une personne et d'en faire le deuil. Les questions étaient ouvertes et, au besoin, accompagnées d'un suivi.

\section{La démographie des participants}

Des renseignements démographiques ont été recueillis par le questionnaire et vérifiés au moment de l'entrevue. 
Tableau 1 : La population

\begin{tabular}{|c|c|c|c|}
\hline & Hommes & Femmes & Transgenres \\
\hline Nombre & 8 & 4 & 3 \\
\hline $\begin{array}{c}\text { Langue } \\
\text { d'interview }\end{array}$ & $\begin{array}{c}2 \text { anglais } \\
6 \text { français }\end{array}$ & $\begin{array}{c}3 \text { anglais } \\
1 \text { français }\end{array}$ & 3 anglais \\
\hline VIH & $\begin{array}{c}\text { 6 positifs } \\
\text { 2 négatifs }\end{array}$ & $\begin{array}{c}4 \text { négatifs } \\
\text { 1 lesbienne } \\
1 \text { bisexuelle } \\
2 \text { hétéro- } \\
\text { sexuelles }\end{array}$ & $\begin{array}{c}2 \text { gais } \\
\text { hétéro- } \\
\text { sexuelle }\end{array}$ \\
\hline
\end{tabular}

\section{Le processus}

Les participants ont été interrogés dans la langue et le lieu de leur choix. Huit personnes l'ont été chez elles et sept dans une salle privée d'un lieu public. Les entrevues étaient enregistrées et transcrites. Chaque participant a signé un formulaire de consentement avant l'entrevue. Chacun recevait vingt dollars en guise de remerciement pour sa participation.

\section{L'analyse}

"Les participants se sont prononcés sur le VIH-sida, sur la mortalité, sur le monde, sur la personne décédée et sur la spiritualité. Des extraits des entrevues sont utilisés pour illustrer chaque thème; au besoin, ils sont traduits de l'anglais. »
L'analyse pour cette étude ne porte que sur les questions s'adressant à la spiritualité et s'effectue selon l'approche de la théorie ancrée (Lincoln \& Guba 1985; Strauss \& Corbin 1990), avec les codes ouverts, axiaux et sélectifs. Chacun de son côté, les deux auteurs ont procédé à toutes les étapes de l'analyse. Des réunions ont permis d'échanger sur les différences dans leurs conclusions respectives.

\section{Les résultats}

Les entrevues portaient sur l'engagement comme thème principal de la spiritualité. Les participants se sont prononcés sur leVIH-sida, sur la mortalité, sur le monde, sur la personne décédée et sur la 
spiritualité. Des extraits des entrevues sont utilisés pour illustrer chaque thème; au besoin, ils sont traduits de l'anglais. L'emploi de pseudonymes permet de protéger l'anonymat des participants.

\section{L'engagement face au VIH-sida}

Les participants se sont engagés face à la maladie et à ses conséquences sur leur vie. Transsexuelle, séropositive depuis 15 ans et âgée de 46 ans au moment de l'entrevue, Chantal travaillait bénévolement, à plein temps, dans une organisation vouée au sida. Elle trouvait que leVIH avait enrichi sa vie : «Je pense que j’ai vécu avec tellement longtemps que si on me disait demain que je n'étais plus séropositive, que je n'avais plus cette maladie, que je serais plus dévastée que quand je l'ai eue. " Chantal était tellement engagée comme sidéenne qu'elle s'identifiait pleinement comme telle.

Rosemary, 52 ans, avait travaillé pendant des années dans une organisation contre le sida. Professionnellement et personnellement, elle s'était engagée face au sida. Elle disait : «Je ne dois rien au sida, mais je dois beaucoup aux gens qui ont contribué à mon bonheur. Pas ma peine, mon bonheur. » Rosemary s'était ainsi engagée afin de pouvoir différencier entre la maladie et les personnes qu'elle connaissait qui en étaient atteintes.

Joseph avait 49 ans au moment de l'entrevue. Séropositif depuis plus de 10 ans, il avait transformé l'acronymeVIH :

"Ce n'est pas que Virus d'immunodéficience humaine, c'est aussi $V$ pour vivre, I pour intensément et $H$ pour l'humain, donc vivre intensément l'humain, et ça me porte depuis le début de mes témoignages, ça me porte certainement depuis 93 ou 94. "

\section{L'engagement face à la mortalité}

Les participants ont souvent exprimé des sentiments vis-à-vis de la mort. Plusieurs ont remarqué qu'ils avaient moins peur de mourir depuis qu'ils avaient pris soin de quelqu'un. Les exemples suivants démontrent comment les gens se sont engagés face à la mortalité. Jean, un séropositif de 39, disait : "Aujourd'hui, on me dirait, je 
"À travers la mort des autres, Jean s'est engagé vis-à-vis de sa propre mortalité." vais mourir dans trois jours, ça ne me dérangerait pas. Parce que j'ai cheminé à travers tout ça. Depuis 1997, j'ai cheminé. J'ai vu d'autres personnes mourir.» À travers la mort des autres, Jean s'est engagé vis-à-vis de sa propre mortalité.

Amy, une femme qui avait très peur de la mort depuis le décès de son père, quand elle était enfant, disait que ce n'était pas possible de bien vivre si on ne faisait pas face à sa propre mortalité. Pour elle, la perte des autres lui a permis de vivre complètement. Michel, un séronégatif dont le partenaire était décédé depuis dix ans au moment de l'entrevue, avait déjà eu très peur de la mort : "Quand ma mère est décédée, ç'a été catastrophique : je pétais les murs, je criais, je la brassais... Ah! Ç’a été l'enfer! »

Depuis la mort de son partenaire, il affirme que la mort ne l'effraie plus, "parce que c'est comme aller quelque part où il $\mathrm{y} a$ déjà quelqu'un. On n'arrive pas dans une maison vide, il y a déjà quelqu'un qui nous reçoit. Donc, on se sent... il y a quelqu'un qui est là.»

\section{L'engagement face au monde}

Ce thème concerne l'engagement des gens dans le monde après la mort d'un être cher. Plusieurs personnes interviewées ont exprimé des idées sur la façon dont le monde devrait se comporter. Ted, 64 ans, séronégatif, avait participé à plus de cinquante équipes qui prenaient soin de personnes atteintes du sida afin de leur permettre de mourir à domicile. Il considérait que ces expériences l'avaient transformé et avaient fait de lui " une meilleure personne ». Il expliquait sa philosophie de la vie : " Aimer et prendre soin. Parce que si tu aimes et tu prends soin, on t'aimera et on prendra soin de toi en retour.»

À travers les expériences, parfois traumatisantes, des gens ont changé pour le mieux. Michel a expliqué comment il a développé plus de compassion pour les autres:

"Moi, je vais regarder par la fenêtre. La journée même du décès, j'ai regardé par la fenêtre et là je vois le vaet-vient des gens, la fourmilière, les petites fourmis qui se promènent et qui font leur travail et sont toutes 
"Donc, on veut aller chercher un peu de compassion, on veut que le monde soit plus compatissant à notre douleur. »
"Dans bien des cas, ils ont affirmé avoir maintenu le dialogue avec les personnes décédées et avoir développé avec elles des rapports différents. " vouées à ça. On est condamnés à ça, ou presque, et je me dis, ça n'a pas de sens. Moi je vis ça, les autres ne le savent pas, là, ce que moi je vis et pourtant d'autres vont le vivre aussi et moi je vais être aussi indifférent qu'eux l'ont été à mon endroit. Donc, on veut aller chercher un peu de compassion, on veut que le monde soit plus compatissant à notre douleur."

Jean et Joseph se sont engagés, chacun de son côté, dans la prévention du sida en présentant des témoignages dans des écoles. Joseph y utilisait sa réinterprétation de l'acronyme VIH. Jean a expliqué ce que cette activité signifiait pour lui :

"J'ai compris qu'on a tous une mission dans la vie, j'ai compris que j'avais un travail à faire et au lieu de rester assis sur mes fesses j'ai décidé de faire de la prévention dans les écoles. Ça fait cinq ans que je fais ça auprès des jeunes... des jeunes et des moins jeunes aussi, partout où je suis demandé. Et ça, c'est une force incroyable. Je sais que je fais du bien auprès des jeunes, que je sauve peut-être, dans une classe, deux vies. Je ne sais pas du tout l'impact. Et en retour, souvent, des jeunes vont m'écrire et vont me dire: "Continue à en parler dans les écoles, ça va peut-être les réveiller". Et ils me confient plein de choses. Et moi ça me donne une force qui me permet de continuer. Je pourrais dire que c'est mon souffle de vie."

\section{L'engagement face à la personne qui est décédée}

Les participants ont beaucoup parlé de la personne disparue, ami ou membre de la famille. Dans bien des cas, ils ont affirmé avoir maintenu le dialogue avec les personnes décédées et avoir développé avec elles des rapports différents. Sarah, une transsexuelle, tenait la photo de son partenaire décédé pour " avoir une conversation " avec lui, parce que, disait-elle, « je crois vraiment qu'[il] n'est pas loin ». Henry, un transgenre de 33 ans et séropositif, avait connu la mort d'un membre de sa famille, de 
"Très peu de temps après la mort de son partenaire, Michel avait l'impression d'une relation renouvelée... il m'accompagne, il est comme là, je n'ai plus physiquement à aller le voir à l'hôpital. » son meilleur ami et de plusieurs autres de ses connaissances. Il a confié : "Je voyage partout dans le monde à des endroits où mes amis auraient voulu aller. Ceux dont j'ai pris soin. Et j'y amène une photo d'eux, je la laisse flotter dans l'eau et je la lâche en disant "tu es là"..."

Au moment de l'entrevue de Gérard, son partenaire était décédé depuis sept ans. Gérard avait 45 ans et, séropositiflui-même, il aimait encore son ami de cœur : «Ton amour ne meurt pas, on dirait que ça... ça nous lie encore ». Dans sa tête, Gérard parlait à son partenaire, qu'il appelait "mon amour » :

"Ça fait quand même sept ans. Moi c'est sûr que mon deuil est fait, le deuil de sa personne, le deuil de [mon ami]. Même si très souvent je lui parle et puis je l'appelle encore "mon amour". Quand je vois de beaux paysages, je lui dis souvent: "regarde comme c'est beau mon amour". Parce que j'ai toujours le sentiment qu'il voit à travers moi, à certains moments. »

Très peu de temps après la mort de son partenaire, Michel avait l'impression d'une relation renouvelée : «Et en sortant de l'hôpital, je me disais : là maintenant, je n'ai plus besoin d'aller à l'hôpital, il m'accompagne, il est comme là, je n'ai plus physiquement à aller le voir à l'hôpital. »

\section{L'engagement face à la spiritualité}

Souvent, les participants proposaient leur propre définition de la spiritualité, faisant fréquemment la distinction entre la spiritualité et la religion. Amy rejetait complètement la religion chrétienne de son enfance et ne croyait plus en Dieu. Malgré tout, elle gardait une Bible chez elle. Autrement dit, elle prenait un peu "de cette religion-ci » et un peu de "cette religion-là ». Avec tous les deuils qu'il a vécus, Henry, un Métis, a développé davantage de spiritualité « en faisant des connexions avec [sa] culture autochtone ".

Lucien, un séropositif âgé de 35 ans, s'était beaucoup engagé dans sa spiritualité et avait réfléchi sur elle et sur la religion : 
"Donc moi dans ma spiritualité c'est que Dieu m'accepte, mais pour autant que je ne fais pas de mal autour de moi."
"[...] je suis spirituel à ma manière, peut-être pas comme les gens l'entendent, parce que j'ai suivi un peu de théologie à l'université... Puis non... Moi,j'ai lâché parce que j'ai ma spiritualité très personnelle... Parce qu'il faut comprendre que la Bible a été écrite par l'homme et non par Dieu. Donc, pour moi,j'en prends et j'en laisse [...] Parce que moi j'ai vu [...] Comme je suis gai, ils disent que c'est péché deux hommes. Ben, en tout cas, c'est drôle, quelques pages avant, Dieu dit “aimez-vous les uns les autres”. Dieu ne t'empêchera pas d'aimer une personne [...] Donc moi dans ma spiritualité c'est que Dieu m'accepte, mais pour autant que je ne fais pas de mal autour de moi."

Joseph, qui avait réinterprété l'acronyme VIH, avait aussi beaucoup réfléchi sur la spiritualité et la religion. Il en était revenu aux symboles religieux et il disait que ça l'aidait de penser que :

"[...] la croix chrétienne a deux sens : il $y$ a le sens évidemment vertical qui est, pour moi en tout cas, le symbole de la relation entre l'homme et Dieu et c'est justement il y a la traverse, le sens horizontal, qui est la relation entre les hommes, entre égaux. »

Et il est revenu à son interprétation du VIH :

"[...] c'est vivre intensément l'humain. Ça inclut une spiritualité. Je pense que les gens qui réussissent dans la vie, même le moine qui est constamment dans une religion et dans la spiritualité, s'il ne développe pas ses qualités humaines il ne pourra pas passer à travers sa vie de moine, ce n'est pas possible. "

\section{Discussion}

Cette étude a donc examiné la spiritualité d'individus ayant pris soin de personnes décédées des complications du sida. Plusieurs des soignants étaient eux-mêmes séropositifs. Leur spiritualité croissait pour inclure l'engagement sur plusieurs plans : face au 
"Les participants ont donné une plus grande signification à leur vie, non seulement à la suite de la mort d'êtres chers, mais aussi en vivant avec la maladie et en faisant face à leur propre mortalité.»

"Plusieurs ont réalisé ce qu'ils avaient appris de la personne disparue et comment ils l'ont intégré à leur propre vie."
VIH-sida, face à la mortalité, face au monde, face à la personne décédée et face à la spiritualité comme telle. L'engagement de ces participants qui avaient vécu un deuil comprenait un processus d'attention (mindfulness) (Langer 1997). L'attention était évidente dans les choix qu'ils avaient faits dans leur vie : comment ils ont choisi de s'impliquer dans la prévention du sida, comment ils voient la mort et comment ils interagissent avec les autres dans le monde.

Les participants ont donné une plus grande signification à leur vie, non seulement à la suite de la mort d'êtres chers, mais aussi en vivant avec la maladie et en faisant face à leur propre mortalité. Plusieurs ont révisé leur perception de la vie en ajustant leurs convictions sur ce qui prévaut et sur ce qui importe dans l'existence. Ce processus de construction d'une nouvelle identité à l'intérieur d'un deuil ou à sa suite gagne de l'importance dans la littérature (Neimeyer 2001). La (re)construction d'un sens à la vie comme réaction à un décès a conduit ces personnes à une perception plus forte d'elles-mêmes dans le monde. De plus, cela leur a permis de s'investir davantage dans la vie et de mieux l'apprécier.

À travers le processus du deuil, ces individus ont développé des rapports spirituels avec les personnes décédées. Ils ont, d'une façon différente, incorporé dans leur vie la personne aimée et adapté leurs liens à cette nouvelle situation (Janzen, Cadell \& Westhues 2004; Klass 2001; Klass, Silverman \& Nickman 1996). Plusieurs ont réalisé ce qu'ils avaient appris de la personne disparue et comment ils l'ont intégré à leur propre vie. Ce faisant, ils ont renforcé le lien spirituel avec la personne décédée, ce qui a eu pour effet d'accroître profondément leur bien-être. Le lien spirituel avec la personne disparue les aide à vivre chaque jour au maximum et a permis d'éliminer chez la plupart la peur de la mort. Pour les participants de cette étude, le lien spirituel à une personne décédée a fortifié leurs convictions spirituelles et leur perception d'eux-mêmes en tant qu'êtres spirituels.

La plupart des participants considéraient la spiritualité comme distincte de la religion. La dichotomie perçue entre les textes religieux qui, d'une part, commandent aux êtres humains de s'aimer 
"La spiritualité individuelle a permis à beaucoup de participants de se sentir intègres face à leur sexualité. Elle a aussi augmenté leur capacité de créer un sens à leur vie et une raison d'être,..."

"Cette étude suggère que la spiritualité pourrait avoir plus d'influence sur la croissance posttraumatique; plus de recherches sont toutefois nécessaires pour explorer la relation de la spiritualité et le phénomène de la croissance posttraumatique." et de s'accepter et qui, d'autre part, condamnent l'homosexualité comme péché, a amené plusieurs participants à se tourner vers une spiritualité individuelle qui leur permet de trouver à l'intérieur de leur personne la force qui viendrait autrement d'une communauté religieuse. La spiritualité individuelle a permis à beaucoup de participants de se sentir intègres face à leur sexualité. Elle a aussi augmenté leur capacité de créer un sens à leur vie et une raison d'être, ce qui leur a permis, même confrontés à leur propre séropositivité et à leur mortalité, de poursuivre leur vie.

Les résultats de cette étude rappellent beaucoup les ressources spirituelles des soignants dans l'étude longitudinale de UCSF (Richards \& Folkman 1997; Richards, Acree \& Folkman 1999; Richards 2001). Contrairement à l'étude américaine, la présente étude pose des questions ayant trait spécifiquement à la spiritualité.

Les thèmes de l'engagement face au VIH-sida, face à la mortalité, face au monde, face à la personne décédée et face à la spiritualité se rapprochent de beaucoup des domaines de la croissance post-traumatique (Cadell 2001; 2003; Cadell, Regehr \& Hemsworth 2003; Tedeschi \& Calhoun 1995; Tedeschi, Park \& Calhoun 1998; Calhoun \& Tedeschi 2001). Le deuil vécu par ces personnes a des aspects qui peuvent traumatiser : que ce soit par la multitude des pertes associées auVIH, ou par la mort d'un être cher après une maladie souvent longue et débilitante. Pour les participants eux-mêmes séropositifs s'ajoute un traumatisme potentiel : observer le déclin d'une personne et entrevoir ce qui pourrait leur arriver à leur tour. Contre toutes ces éventualités, les participants ont démontré des résultats positifs semblables à la croissance post-traumatique (Tedeschi \& Calhoun 1996). Cette étude suggère que la spiritualité pourrait avoir plus d'influence sur la croissance post-traumatique; plus de recherches sont toutefois nécessaires pour explorer la relation de la spiritualité et le phénomène de la croissance post-traumatique. 


\section{Conclusion}

Tout au long de notre étude, la spiritualité s'est exprimée à travers les témoignages de quinze individus qui, sous différentes formes d'engagement, ont pris soin de personnes atteintes du sida. Face au deuil engendré par cette maladie, la spiritualité a été mentionnée comme un facteur important d'adaptation, permettant aux soignants de poursuivre leur vie et, dans certains cas, d'assumer leur propre séropositivité. Le renforcement de leur spiritualité les a beaucoup aidés à redonner à leur vie le sens que le VIH-sida lui avait enlevé.

\section{Bibliographie}

BIGELOW, G. \& Hollinger, J. (1996). "Grief and AIDS: Surviving Catastrophic Multiple Loss”, The Hospice Journal, 11(4), 83-96.

CADELL, S. (2001). "Post-traumatic Growth in HIV/AIDS Caregivers in Quebec”, Canadian Social Work, 3(1), 86-94.

CADELL, S. (2003) “Trauma and Growth in Canadian Carers”, AIDS Care, 15(5), 639-648.

CADELL, S., Regehr, C. \& Hemsworth, D. (2003). "Factors Contributing to Post-traumatic Growth: A Proposed Structural Equation Model”, American Journal of Orthopsychiatry, 73(3), 279-287.

CALHOUN,L.G. \& Tedeschi, R.G. (2001) “Post-traumatic Growth:The Positive Lessons of Loss”, In R.A. Neimeyer (Ed.), Meaning Reconstruction and the Experience of Loss (pp. 157-172), Washington, DC: American Psychological Association.

CANDA, E. \& Furman, L.D. (1999). Spiritual Diversity in Social Work Practice: the Heart of Helping. New York: Free Press.

FOLKMAN, S. (1997). "Positive Psychological States and Coping with Severe Stress”, Social Science and Medicine, 45(8), 1207-1221.

FOLKMAN, S., Chesney, M., Collette, L., Boccellari, A. \& Cooke, M. (1996). "Postbereavement Depressive Mood and its Prebereavement Predictors in HIV+ and HIV- Gay Men”, Journal of Personality and Social Psychology, 70(2), 336-348.

FOLKMAN, S., Chesney, M.A., Cooke, M., Boccellari, A. \& Collette, L. (1994). “Caregiver Burden in HIV-Positive and HIV-Negative Partners of Men with AIDS", Journal of Consulting and Clinical Psychology, 62(4), 746-756.

FOLKMAN, S. \& Moskowitz,.J.T. (2000). "Stress, Positive Emotion, and Coping" Current Directions in Psycological Science, 9(4), 115-118.

FOLKMAN, S., Moskowitz, J.T., Ozer, E.M. \& Park, C.L. (1997). “Positive Meaningful Events and Coping in the Context of HIV/AIDS”, In Gottlieb, B.H. (Ed.), Coping with Chronic Stress, New York: Plenum Press. 
GLUHOSKI,V.L., Fishman, B. \& Perry, S.W. (1997b). “Moderators of Bereavement Distress in a Gay Male Sample”, Personality and Individual Differences, 23(5), 761-767.

GOODKIN, K., Blaney, N.T., Tuttle, R.S., Nelson, R.H., Baldewicz, T., Kumar, M., Fletcher, M.A., Leeds, B. \& Feaster, D.J. (1996). "Bereavement and HIV Infection”, International Review of Psychiatry, 8, 201-216.

HARDY, R.P. (1982), “Christian Spirituality Today: Notes on its Meaning”, Spiritual Life, 28, 151159.

HARDY, R.P. (1998). Loving Men: Gay Partners, Spirituality, and AIDS, New York: The Continuum Publishing Company.

HELMINIAK, D.A. (1995). "Non-Religious Lesbians and Gays Facing AIDS:A Fully Psychological Approach to Spirituality”, Pastoral Psychology. 43(5), 301-318.

HOUSEMAN, C. \& Pheifer, W.G. (1988). "Potential for Unresolved Grief in Survivors of Persons with AIDS", Archives of Psychiatric Nursing. 2(5), 296-301.

JACOBS, C. (1997). "On Spirituality and Social Work Practice”, Smith College Studies in Social Work. 67(2), 171-175.

JANZEN, L.M., Cadell, S. \& Westhues,A. (2004). "Dealing with the Sudden Death of a Child:Advice to Professionals from Parents", Omega: Journal of Death and Dying. 48(2), 175-190.

KLASS, D. (2001). "The Inner Representation of the Dead Child in the Psychic and Social Narratives of Beraeved Parents”, In R.A. Neimeyer (Ed.), Meaning Reconstruction and the Experience of Loss (pp. 77-94), Washington, DC: American Psychological Association.

KLASS, D., Silverman, P.R. \& Nickman, S.L. (1996). Continuing Bonds: New Understandings of Grief, Washington, DC: Taylor \& Francis.

KOZENY, G. (2004). "Dancing with Dogma:The Fine Line between Religion and Spirituality", Communities, 124, 72-73.

LANGER, E.J. (1997). The Power of Mindful Learning, Reading, MA:Addison-Wesley Publishing.

LAZARUS, R.S. \& Folkman, S. (1984). Stress, Appraisal, and Coping, New York: Springer Publishing Company.

LINCOLN,Y.S. \& Guba, E.G. (1985). Naturalistic Inquiry, Beverly Hills, CA: Sage Publications.

MARTIN, J.L. \& Dean, L. (1993). "Effects of AIDS-Related Bereavement and HIV-Related Illness on Psychological Distress Among Gay Men:A 7-Year Longitudinal Study, 1985-1991”, Journal of Consulting and Clinical Psychology. 61(1), 94-103.

MOSKOWITZ, J.T., Folkman, S., Collette, L. \& Vittinghoff, E. (1996), “Coping and Mood during AIDS-Related Caregiving and Bereavement”, Annals of Behavioral Medicine. 18(1), 49-57.

NEIMEYER, R.A. (2001). "Meaning Reconstruction and Loss", In R.A. Neimeyer (Ed.), Meaning Reconstruction and the Experience of Loss (pp. 1-9), Washington, DC: American Psychological Association.

NEUGEBAUER, R., Rabkin, J.G., Williams, J.B.W., Remien, R.H., Goetz, R. \& Gorman, J.M. (1992). "Bereavement Reactions Among Homosexual Men Experiencing Multiple Losses in the AIDS Epidemic”, American Journal of Psychiatry, 149(10), 1374-1379.

NORD, D. (1996a). "Assessing the Negative Effects of Multiple AIDS-Related Loss on the Gay Individual and Community", Journal of Gay and Lesbian Social Services. 4(3), 1-34.

NORD, D. (1996b). "Issues and Implications in the Counseling of Survivors of Multiple AIDSRelated Loss", Death Studies. 20(4), 389-413. 
NORD, D. (1997). Multiple AIDS-Related Loss:A Handbook for Understanding and Surviving a Perpetual Fall, Washington, D.C.: Taylor \& Francis.

NORD, D. (1998). “Traumatization in Survivors of Multiple AIDS-Related Loss", Omega: Journal of Death and Dying, 37(3), 215-240.

PARGAMENT, K.I. \& Park, C.L. (1995). "Merely a Defense? The Variety of Religious Means and Ends”, Journal of Social Issues, 51(2), 13-32.

RICHARDS, T.A. (2001). "Spiritual Resources Following a Partner's Death from AIDS”, In R.A. Neimeyer (Ed.), Meaning Reconstruction and the Experience of Loss (pp. 173-190), Washington, DC: American Psychological Association.

RICHARDS, T.A., Acree, M. \& Folkman, S. (1999). "Spiritual Aspects of Loss among Partners of Men with AIDS: Postbereavement Follow-up", Death Studies, 23(2), 105-127.

RICHARDS, T.A. \& Folkman, S. (1997). "Spiritual Aspects of Loss at the Time of a Partner's Death from AIDS", Death Studies, 21(6), 527-552.

SHERNOFF, M. (1995). "Reflections on Living with AIDS", Journal of Gay and Lesbian Social Services, 3(4), 83-89.

SHERNOFF, M. (1997a). "Conclusion: Mental Health Considerations of Gay Widowers”, Journal of Gay and Lesbian Social Services, 7(2), 137-155.

SHERNOFF, M. (1997b). "Do You Have a Partner?”, Journal of Gay and Lesbian Social Services, 7(2), $15-28$.

SIKKEMA, K.J., Kalichman, S.C., Kelly, J.A. \& Koob, J.J. (1995). “Group Intervention to Improve Coping with AIDS-Related Bereavement: Model Development and an Illustrative Clinical Example", AIDS Care, 7(4), 463-475.

STRAUSS, A. \& Corbin, J. (1990). Basics of Qualitative Research: Grounded Theory Procedures and Techniques, Newbury Park, CA: Sage Publications.

TEDESCHI, R.G. \& Calhoun, L.G. (1995). Trauma and Transformation: Growing in the Aftermath of Suffering, Thousand Oaks, CA: Sage Publications.

TEDESCHI, R.G. \& Calhoun, L.G. (1996). “The Post-traumatic Growth Inventory: Measuring the Positive Legacy of Trauma”, Journal of Traumatic Stress, 9(3), 455-471.

TEDESCHI, R.G., Park, C.L. \& Calhoun, L.G. (1998). Post-traumatic Growth: Positive Changes in the Aftermath of Crisis, Mahwah, NJ: Lawrence Erlbaum Associates.

WARDLAW, L.A. (1994). "Sustaining Informal Caregivers for Persons with AIDS", Families in Society, 75(6), 373-384.

WRUBEL, J. \& Folkman, S. (1997). "What Informal Caregivers Actually Do:The Caregiving Skills of Partners of Men with AIDS”, AIDS Care, 9(6), 691-706.

\section{Note}

1 Cette étude a été subventionnée par une bourse d'études supérieures du Conseil de recherche en sciences humaines du Canada. Les auteurs voudraient remercier Jacques Ouellette pour l'inspiration. 


\section{Annexe : Le guide de l'entretien}

Parlez-nous de ce que vous avez vécu en accompagnant quelqu'un qui se mourait du sida?

Cette expérience vous a-t-elle changé? Comment?

Qu'est-ce qui a aidé dans le processus de changement?

Qu'est-ce qui n'a pas aidé?

Est-ce que la spiritualité vous a aidé?

Y a-t-il autre chose qui vous a aidé? 